\title{
Association between Thyroid Hormones, Apolipoprotein E, and Cognitive Function among Cognitively-Normal Elderly Dwellers
}

\author{
Beomjun Kim and Seok Woo Moon $\bowtie$ \\ Department of Psychiatry, Research Institute of Medical Science, Konkuk University Medical School, Chungju, Republic of Korea
}

\begin{abstract}
Objective The correlation among the thyroid-related hormones, Apolipoprotein E $\varepsilon 4$ (APOE $\varepsilon 4$ ) and cognitive function has been reported despite controversial results. This study was designed to investigate this correlation among cognitively-normal elderly dwellers.

Methods This study assessed 507 cognitively normal individuals aged over 60 who underwent comprehensive hematological and neuropsychological assessments including the quantification of serum free thyroxine and thyroid stimulating hormone (TSH) as well as the Korean version of the Consortium Establish a Registry for Alzheimer's disease. The Korean version of Geriatric Depression Scale was also employed to evaluate the severity of depression. Age, gender, education, and the presence of APOE $\varepsilon 4$ were taken into account as covariates.

Results There was a significant positive association between verbal fluency test (VFT), Word List Memory Test (WLMT), and Word List Recall Test (WLRT) score and serum TSH levels ( $\mathrm{p}=0.007,0.031$, and 0.023 respectively). The further analysis adding the interaction between APOE $\varepsilon 4$ and TSH level, however, revealed only VFT score was significantly influenced by this interaction ( $\mathrm{p}=0.026)$.

Conclusion Lower serum TSH levels had impacts on both semantic memory (VFT) and episodic memory (WLMT, WLRT) among cognitively-normal elderly, whereas the interaction of TSH and APOE $\varepsilon 4$ influenced only the task of semantic memory (VFT) in this group.

Psychiatry Investig 2020;17(10):1006-1012
\end{abstract}

Key Words Thyroid hormones, APOE, Cognitive function, Memory, Aged.

\section{INTRODUCTION}

Alzheimer's disease $(\mathrm{AD})$ has been regarded as the most common cause of neurodegenerative cognitive disorders. ${ }^{1,2}$ The absence of specific biomarkers for the definite diagnosis of $\mathrm{AD}$ causes $\mathrm{AD}$ to remain defined as a disease with certain clinical manifestations. ${ }^{2,3}$ Major approaches for managing AD, such as acetylcholinesterase inhibitors and memantin, are not only symptomatic measures, but also unlikely to affect a late stage of $\mathrm{AD}^{3,4}$ This situation implies that the early detection of its risk factors and subsequently early interventions thus far remain one of the most practical treatment options. ${ }^{3,4}$

Alteration of the thyroid hormonal state has been reported

Received: May 18, 2020 Revised: July 20, 2020

Accepted: August 23, 2020

$\triangle$ Correspondence: Seok Woo Moon, MD, PhD

Department of Psychiatry, Konkuk University Chungju Hospital, 82 Gukwondaero, Chungju 27354, Republic of Korea

Tel: +82-43-840-8990, Fax: +82-43-857-1380, E-mail: hessem@kku.ac.kr

(c) This is an Open Access article distributed under the terms of the Creative Commons Attribution Non-Commercial License (https://creativecommons.org/licenses/by$\mathrm{nc} / 4.0$ ) which permits unrestricted non-commercial use, distribution, and reproduction in any medium, provided the original work is properly cited. to constitute a risk factor for developing dementia at a later stage of life. ${ }^{5,6}$ The Apolipoprotein E (APOE) gene has also been hypothesized to be a risk factor for late-onset $\mathrm{AD}^{7,8}$ As a result, research has been conducted to elucidate how these risk factors are correlated with cognitive function, since cognitive impairment was reported to be one of initial symptoms of AD. ${ }^{9}$

Thyroid hormones are essential for the development and metabolism of the brain, ${ }^{10}$ ranging from cellular- to geneticlevel processes. ${ }^{11-13}$ Indeed, epidemiologic studies have identified subclinical hyperthyroidism or a decreased level in TSH was significantly associated with cognitive impairment, ${ }^{14-16}$ while others have failed to ensure this correlation. ${ }^{17-19}$ Similar inconsistent results have also been identified in specific cognitive domains primarily affected by thyroid function. Recent publications, for instance, revealed that lower TSH levels profoundly influenced executive function rather than memory, ${ }^{20,21}$ whereas other investigators did not reproduce this outcome. ${ }^{18,22}$ These contrasting outcomes might be ascribed to characteristics of sample, types of thyroid-related hormones, and neuropsychological tools that each study had implemented.

Aside from TSH, the APOE gene has been also investigated 
in light of its influence on cognitive performance. Even though etiologies of $\mathrm{AD}$ have not been fully identified, carriers of the APOE $\varepsilon 4$ allele are more predisposed to developing AD in a dose-dependent manner. ${ }^{23}$ This allele has been reported to be correlated to cognitive impairment across cognitively-normal $(\mathrm{CN})$ and cognitively-impaired elderly over the age of $60{ }^{7,23,24}$ In particular, prior studies have revealed that the $\varepsilon 4$ allele of APOE was correlated with poor memory performance in nondemented participants, ${ }^{25}$ although others have not shown any significant association between the $\varepsilon 4$ allele and decreased scores in memory tests among this group..$^{23,25}$

Incorporating with aforementioned research on $\mathrm{TSH}$, these studies regarding the influence of APOE on memory performance lend a perspective concerning the association between thyroid hormones and APOE in the memory system. Previous studies relevant to these variables have tended to conducted across individuals with demented or mild cognitive impairment (MCI), primarily because these parameters are believed to be correlated with developing $\mathrm{AD}$ and one of initial symptoms of $\mathrm{AD}$ have been reported to be memory deficit. With the consideration of these two parameters as risk factors of $\mathrm{AD}$, our study was designed to scrutinize their interplay in far earlier stages of MCI which can refer to a premorbid stage of $\mathrm{AD}$, that is, cognitively-healthy state of elderly population. In light of these perspectives, our study aimed at investigating the hypothesis that these two factors and their interaction are more associated with memory performance than other domains in cognition among $\mathrm{CN}$ individuals.

\section{METHODS}

\section{Selection criteria of participants}

Participants were recruited from 2011 to 2018 as a part of Dementia Screening Program organized by Korean Ministry of Health and Welfare, all of whom were elderly over the age of 60 and dwelled in Chungcheongbuk-do, Korea. After being evaluated by Korean version of the Consortium Establish a Registry for Alzheimer's Disease (CERAD-K) and Clinical Dementia Rating (CDR), subjects whose CDR score was zero were only included in the study. Modified Hachinski Ischemia Scale was also calculated by a senior psychiatrist so that individuals whose score was below 3 could enter the study. Based on Diagnostic Statistical Manual of Mental Disorders, fourth edition, test revision (DSM-IV_TR), only subjects not diagnosed with major depressive disorder were included. In order to minimize confounding factors, we excluded participants who manifested any sign of altered consciousness, as well as severe neurovascular pathology and neurological diseases. Moreover, we did not employ data from individuals who not only had medical illnesses including thyroid diseases, but also were receiving medications which may be able to alter cognitive function. All participants underwent hematologic tests to measure the serum concentration of free thyroxine (fT4) and $\mathrm{TSH}$, and the identification of the APOE $\varepsilon 4$ after obtaining an informed consent. This research was approved by Institutional Review Board of Konkuk University Chungju Hospital (KUCH 2019-12-044) and all researchers abided by its ethical codes of conduct.

\section{Identification of APOE}

Whole blood cell was obtained via an ethylenediaminetetraacetic acid tube, white blood cell being segregated to extract Genomic DNA. It was analyzed through Polymerase chain reaction-restriction fragment length polymorphism to identify the APOE $\& 4$ allele. $^{26}$

\section{Serum fT4, serum TSH and classification of thyroid function}

Subjects were encouraged to visit clinics in the morning while maintaining a fasting state wherever possible, in order to collect serologic samples. Serum fT4 and TSH were assessed using a chemiluminescent immunoassay in the department of laboratory medicine, Konkuk university Chungju hospital. Laboratory reference ranges of serum $\mathrm{fT} 4$ and TSH record $0.70-1.48 \mathrm{ng} / \mathrm{dL}$ and $0.35-4.93 \mu \mathrm{IU} / \mathrm{mL}$ respectively. For the convenience of statistical analysis, TSH levels were evenly divided into three tertiles, resulting in tertile $1(<2.0)$, tertile 2 $(2.0-3.0)$, and tertile 3 (>3.0). In detail, in terms of a number of subjects belonging to each tertile, this numerical-based tertiles led to relatively more even-distribution rather than a group based on serum levels of hyper-, eu-, and hypothyroid states.

\section{Neuropsychological assessment tool}

Cognitive function was assessed by CERAD- $\mathrm{K}^{27}$ comprising Verbal Fluency Test (VFT), Modified Boston Naming Test (mBNT), Mini-Mental State Examination in the Korean version of CERAD assessment packet (MMSE-KC), Word List Memory Test (WLMT), Construction Praxis Test (CPT), Word List Recall Test (WLRT), Word List Recognition Test (WLRcT), and Construction Recall Test (CRT). It was conducted by research nurses who were trained for this study and supervised by a senior researcher and psychiatrist.

\section{Clinical Dementia Rating}

A psychiatrist recorded CDR scales by interviewing family members of participants regarding six domains constituting $\mathrm{CDR}$, including memory, orientation, judgment and problem solving, community affairs, home and hobbies, and personal care. Subjects rated $\mathrm{CDR}=0$ were only included in the study. 


\section{Depression}

Korean version of Geriatric Depression Scale (GDS-K) consisting of 30 questions was employed as a self-reporting format to assess depression. Illiterate subjects completed the form by answering original questions read by a research nurse. This scale was utilized to exclude the impact of depression on results.

\section{Statistical analyses}

Demographic profiles and clinical characteristics were compared via $\chi^{2}$ and t-test depending on categorical and continuous features of variables respectively. Further analysis was performed by Multivariate Analysis of Variance (MANOVA).

We divided the TSH level into three strata to identify the TSH level that primarily affects neuropsychological test scores. The TSH tertiles and APOE $\varepsilon 4$ positivity were used as fixed factors, whereas age, education, and gender were treated as covariances to make Model I. An interaction effect between TSH tertiles and APOE $\varepsilon 4$ was explored while controlling for confounding factors which were covariances to make Model II. The difference in mean neuropsychological test scores was investigated among TSH strata using the general linear model (GLM) analysis with post-hoc tests using the least significant difference (LSD) method. Data were analyzed using SPSS 24.0 software (IBM Corp., Armonk, NY, USA).

\section{RESULTS}

The demographic profile revealed subjects mean age and the average year of education recorded 74.48 and 6.81 respectively. The proportion of participants in each tertile was composed of $26.2 \%$ (Tertile 1), 30.0\% (Tertile 2), and $43.6 \%$ (Tertile $3)$. The APOE $\varepsilon 4$ was detected in 100 participants (19.7\%). The analyses of clinical characteristics of subjects based on the TSH concentration demonstrated no significant correlation of TSH level with each characteristics excluding fT4 level. Particularly, GDS-K was also found to be not correlated with TSH level among CN participants (Table 1).

The analyses of neuropsychological variances revealed the presence of the APOE $\varepsilon 4$ gene was significantly associated with the WLMT and WLRT scores, while the increased level of TSH was also correlated with the elevated score of VFT as well as those variances (Table 2, Model I). The further investigation of individual neuropsychological components in relation to the TSH level by introducing the interaction effect of APOE $\varepsilon 4$ discovered that VFT recorded the only variance significantly associated with this interaction $(\mathrm{p}=0.026)$. On the contrary, there was no significant association between fT4 levels and scores of neuropsychological tests (Table 3).

Figure 1 illustrated this outcome in which the existence of APOE $\varepsilon 4$ significantly tended to decrease the VFT score despite the increase of the TSH level among participants, while the absence of APOE $\varepsilon 4$ led to the elevation of this score accordingly.

\section{DISCUSSION}

The major findings of this present study revealed that the main effect of the TSH level was significantly correlated with the VFT, WLMT, and WLRT among CN elderly individuals. The further analysis resulted in the identification of a significant interaction between APOE $\varepsilon 4$ and the TSH concentration in the VFT. As depicted in the Figure 1, this implies that the elevated level of TSH could not contribute to higher score of VFT in the existence of the APOE $\varepsilon 4$ allele. Incorporating with the fact that VFT task of CERAD-K is designed to assess semantic memory (SM) as opposed to WLMT and WLRT representing episodic memory (EM), this could imply that the interaction between the APOE $\varepsilon 4$ and TSH significantly affected SM of CN subjects, in comparison with the episodic component of memory.

Since the alteration of a TSH level tends to occur in accordance with aging process and cognitive decline often accompanies aging concomitantly, ${ }^{15,28}$ the correlation between TSH and

Table 1. Clinical characteristics of subjects by thyroid stimulating hormone (TSH) level status ${ }^{\dagger}$

\begin{tabular}{|c|c|c|c|c|c|}
\hline & Total & TSH $1(<2.0 \mu \mathrm{IU} / \mathrm{mL})$ & TSH $2(2.0-3.0 \mu \mathrm{IU} / \mathrm{mL})$ & TSH $3(>3.0 \mu \mathrm{IU} / \mathrm{mL})$ & p-value \\
\hline Participants, N (\%) & $507(100)$ & $134(26.2)$ & $152(30.0)$ & $221(43.6)$ & \\
\hline $\mathrm{TSH}(\mathrm{mIU} / \mathrm{L}), \mathrm{M}(\mathrm{SD})^{\ddagger}$ & $2.180(0.82)$ & $1.193(0.386)$ & $2.284(0.371)$ & $4.912(2.956)$ & $0.001^{*}$ \\
\hline Age, years, M (SD) & $74.48(7.957)$ & $75.16(7.693)$ & $73.61(7.763)$ & $74.67(8.225)$ & 0.231 \\
\hline Gender, male, $\mathrm{N}(\%)$ & $275(39.3)$ & $69(51.5)$ & $92(60.5)$ & $110(49.8)$ & 0.107 \\
\hline Years of education, $M(\mathrm{SD})$ & $6.81(3.477)$ & $6.24(3.648)$ & $6.54(3.018)$ & $7.34(4.648)$ & 0.143 \\
\hline Free T4 (ug/dL) & $1.069(0.259)$ & $1.133(0.256)$ & $1.059(0.225)$ & $1.037(0.277)$ & $0.003^{*}$ \\
\hline APOE $\varepsilon 4, N(\%)$ & $100(19.7)$ & $26(19.4)$ & $33(21.7)$ & $41(18.6)$ & 0.749 \\
\hline GDS-K total score, $\mathrm{M}$ (SD) & $9.97(6.906)$ & $10.62(7.077)$ & $9.78(6.669)$ & $9.70(6.965)$ & 0.440 \\
\hline
\end{tabular}

${ }^{*} \mathrm{p}<0.05$, the statistics were analyzed using analysis of variance (ANOVA), ${ }^{\ddagger} \mathrm{M}$ (SD): mean (standard deviation). TSH: thyroid stimulating hormone, T4: thyroxine, APOE: apolipoprotein E, GDS-K: Korean version of Geriatric Depression Scale 
Table 2. Interactions between APOE and TSH level associated with Neuropsychological test scores ${ }^{\dagger}$

\begin{tabular}{|c|c|c|c|c|c|c|c|c|c|c|c|c|c|c|c|}
\hline & \multicolumn{5}{|c|}{ APOE $\varepsilon 4$ status } & \multicolumn{5}{|c|}{ TSH level } & \multicolumn{5}{|c|}{$\begin{array}{c}\text { APOE } \varepsilon 4 \text { status*TSH level } \\
\text { (interaction term) }\end{array}$} \\
\hline & Type III SS & $\mathrm{df}$ & MS & $\mathrm{F}$ & $\mathrm{p}$ value & Type III SS & $\mathrm{df}$ & MS & $\mathrm{F}$ & $\mathrm{p}$ value & Type III SS & $\mathrm{df}$ & MS & $\mathrm{F}$ & $\mathrm{p}$ value \\
\hline \multicolumn{16}{|l|}{ Model I } \\
\hline VFT & 7.968 & 1 & 7.968 & 0.710 & 0.400 & 112.086 & 2 & 56.043 & 4.991 & $0.007^{*}$ & & & & & \\
\hline BNT & 2.797 & 1 & 2.797 & 0.528 & 0.468 & 6.634 & 2 & 3.317 & 0.626 & 0.535 & & & & & \\
\hline WLMT & 44.248 & 1 & 44.248 & 4.000 & $0.046^{\dagger}$ & 77.273 & 2 & 38.637 & 3.493 & $0.031^{*}$ & & & & & \\
\hline $\mathrm{CPT}$ & 0.429 & 1 & 0.429 & 0.262 & 0.609 & 2.809 & 2 & 1.405 & 0.860 & 0.424 & & & & & \\
\hline WLRT & 17.046 & 1 & 17.046 & 6.108 & $0.014^{\dagger}$ & 21.307 & 2 & 10.653 & 3.817 & $0.023^{\dagger}$ & & & & & \\
\hline WLRcT & 3.276 & 1 & 3.276 & 1.509 & 0.220 & 6.303 & 2 & 3.152 & 1.452 & 0.235 & & & & & \\
\hline CRT & 4.345 & 1 & 4.345 & 0.694 & 0.405 & 19.369 & 2 & 9.685 & 1.547 & 0.214 & & & & & \\
\hline \multicolumn{16}{|l|}{ Model II } \\
\hline VFT & 20.186 & 1 & 20.186 & 1.817 & 0.178 & 146.277 & 2 & 73.139 & 6.584 & $0.002^{*}$ & 81.999 & 2 & 41.000 & 3.691 & $0.026^{*}$ \\
\hline BNT & 2.239 & 1 & 2.239 & 0.421 & 0.517 & 7.392 & 2 & 3.696 & 0.695 & 0.499 & 0.875 & 2 & 0.438 & 0.082 & 0.921 \\
\hline WLMT & 50.122 & 1 & 50.122 & 4.547 & $0.033^{\dagger}$ & 56.867 & 2 & 28.434 & 2.579 & 0.077 & 41.059 & 2 & 20.530 & 1.862 & 0.156 \\
\hline $\mathrm{CPT}$ & 0.294 & 1 & 0.294 & 0.180 & 0.671 & 1.033 & 2 & 0.516 & 0.317 & 0.729 & 4.000 & 2 & 2.000 & 1.226 & 0.294 \\
\hline WLRT & 19.79 & 1 & 19.790 & 7.101 & $0.008^{\dagger}$ & 11.338 & 2 & 5.669 & 2.034 & 0.132 & 7.578 & 2 & 3.789 & 1.360 & 0.258 \\
\hline WLRcT & 4.429 & 1 & 4.429 & 2.041 & 0.154 & 5.324 & 2 & 2.662 & 1.227 & 0.294 & 4.892 & 2 & 2.446 & 1.127 & 0.325 \\
\hline CRT & 4.782 & 1 & 4.782 & 0.761 & 0.383 & 17.927 & 2 & 8.963 & 1.426 & 0.241 & 0.992 & 2 & 0.496 & 0.079 & 0.924 \\
\hline
\end{tabular}

${ }^{*} \mathrm{p}<0.05$, †general linear model (GLM) analysis Model I: APOE and TSH level, Model II: Model I+APOE*TSH level (interaction term). Age, gender, education and Korean version of Geriatric Depression Scale (GDS-K) adjusted for multiple analysis of covariance (MANOVA). APOE: apolipoprotein E, TSH: thyroid stimulating hormone, SS: square sum, MS: mean squre, VFT: verbal fluency test, mBNT: modified Boston naming test, WLMT: word list memory test, CPT: construction praxis test, WLRT: word list recall test, WLRcT: word list recognition test, CRT: construction recall test

Table 3. Neuropsychological test scores associated with free T4 level (3 strata)*

\begin{tabular}{lccccc}
\hline & Type III SS & df & MS & F & p value \\
\hline VFT & 23.385 & 2 & 11.693 & 2.222 & 0.110 \\
BNT & 7.379 & 2 & 3.689 & 0.322 & 0.725 \\
WLMT & 4.748 & 2 & 2.374 & 1.092 & 0.336 \\
CPT & 1.52 & 2 & 0.76 & 0.465 & 0.629 \\
WLRT & 12.629 & 2 & 6.314 & 1.006 & 0.366 \\
WLRcT & 4.97 & 2 & 2.485 & 0.222 & 0.801 \\
CRT & 3.02 & 2 & 1.51 & 0.534 & 0.587 \\
\hline
\end{tabular}

*general linear model (GLM) analysis. Age, gender, education and Korean version of Geriatric Depression Scale (GDS-K) adjusted for multiple analysis of covariance (MANOVA). SS: square sum, MS: mean squre, VFT: verbal fluency test, mBNT: modified Boston naming test, WLMT: word list memory test, CPT: construction praxis test, WLRT: word list recall test, WLRcT: word list recognition test, CRT: construction recall test

alteration in cognition has been studied. ${ }^{14}$ Indeed, one study demonstrated low concentration of TSH was a risk factor of developing dementia, ${ }^{28}$ and it was also reported that low TSH was significantly associated with cognitive decline among the elderly population, ${ }^{29}$ which corresponds to our outcomes.

The mechanism underlying the relation between TSH and cognitive function remains uncertain. Experimental research revealed thyroid hormones play a part in the development of glial cells, proper synaptogenesis and myelination of nerve cells. ${ }^{30,31}$ Elevated TSH in response to decreased thyroid hormones can elicit oxidative stress and subsequently trigger apoptotic neuronal cell death. ${ }^{15,29}$ Additionally, as thyrotropin-releasing hormone (TRH) has been reported to modulate in acetylcholine synthesis and release, a decreased level of TRH induced by elevated secretion of thyroid hormones may result in disturbance in brain acetylcholine metabolism, which is believed to be related to dementia. ${ }^{15,29}$ Particularly, TSH has been reported to be associated with regional cerebral blood flow in the bilateral temporal and hippocampal areas, ${ }^{32}$ which have been thought to contribute to the formation of explicit memory. ${ }^{32,33}$ Similarly, our study found the positive correlation between the concentration of TSH and scores in VFT, WLMT, and WLRT. A similar pattern was also illustrated in an epidemiological study reporting that decrease in TSH was related to decline in verbal fluency among $\mathrm{CN}$ elderly participants aged over $75 .^{22}$

Our findings indicated the presence of the $\varepsilon 4$ allele could affect memory performance across $\mathrm{CN}$ elderly. $\mathrm{CN}$ middle-aged individuals carrying APOE $\varepsilon 4$ allele were reported to show diminished volume of hippocampus, alongside decreased cerebral metabolic rates of glucose in both parietotemporal, pre- 

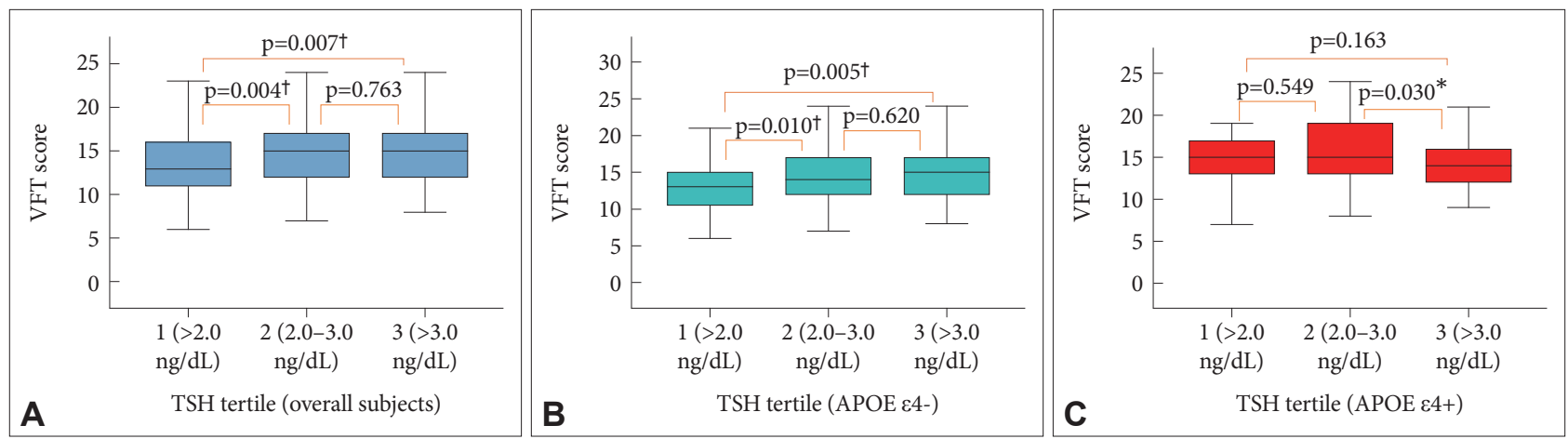

Figure 1. Moderating effects of APOE $\varepsilon 4$ allele on the relationships between TSH level and VFT. A: Overall. B: APOE $\varepsilon 4$ (-). C: APOE $\varepsilon 4$ (+). ${ }^{*} p<0.05,{ }^{\dagger} p<0.01$. VFT: verbal fluency test, TSH: thyroid stimulating hormone, APOE: apolipoprotein E.

frontal and posterior cingulate areas using FDG PET. ${ }^{32}$ These areas have been well-established to be involved in memory performance. As implied in this connection, a recent study reported older participants carrying the APOE $\varepsilon 4$ allele demonstrated poor performance in VFT, while this correlation did not exist after the exclusion of cognitively-impaired participants. ${ }^{8}$ Other studies illustrated that APOE $\varepsilon 4$ allele influenced an episodic memory test as compared with a semantic counterpart. ${ }^{34}$ This is consistent with our results displaying the significant association between the presence of the APOE $\varepsilon 4$ allele and scores in WLMT and WLRT. Interestingly, further analysis illustrated only VFT was significantly influenced by the interaction of APOE and TSH, as opposed to WLMT and WLRT. In other words, our results demonstrated that VFT representing SM was negatively influenced by the concurrent presence of the APOE $\varepsilon 4$ and TSH, while the existence of APOE without its interaction with TSH did not significantly affect the VFT score. In light of the well-acknowledged association between the $\varepsilon 4$ allele and $\mathrm{AD}$, our findings are supported by a prior study illustrating significant impairment in $\mathrm{SM}$ relative to $\mathrm{EM}$ among $\mathrm{AD}$ patients. ${ }^{35}$ Another recent study also revealed potential interplay between the $\varepsilon 4$ allele and TSH regarding cognitive function as implied in our study. ${ }^{36}$

One possibility concerning this apparent interaction between TSH and APOE could be attributed to their interplay affecting $\beta$-amyloid which plays an essential role in $\mathrm{AD}$ pathogenesis. ${ }^{14}$ The $\varepsilon 4$ allele has been thought to be involved in the accumulation of neurotoxic deposits related to $\beta$-amyloid, toxicity of which is believed to have detrimental effects on memory formation process. Furthermore, thyroid hormones are thought to not only regulate the precursor of $\beta$-amyloid protein, but also influence the brain metabolism and the modulation of APOE gene expression in the brain. ${ }^{21,37}$ Both APOE and alteration of TSH is also believed to influence hyperphosphorylation of tau protein involved in the pathogenesis of $\mathrm{AD},{ }^{14,36}$ which sheds additional light on likelihoods of the interaction of these parameters.
On the other hand, this phenomenon can also be explained by characteristics of the neuropsychological test used in our study. The VFT of our study needs participants to generate names as many as possible after listening to a cue within oneminute-time frame, which means it requires both effortful retrieval and psychomotor speed pertaining to executive function, as well as semantic processing. ${ }^{33,38,39}$ In other words, the score of VFT depending on this method can represent combined effects of these two categories of cognitive function. On the contrary, total scores of WLMT and WLRT did not include interference words generated by participants, which did not exist in original word lists. These scores, therefore, relatively reflected pure episodic components of memory without executive abilities. This could lead to discrepant results between VFT and other tests. This could be supported by a recent study demonstrating that the $\varepsilon 4$ allele carriers who were cognitively intact recorded significant decline in executive function, while no statistical association between APOE and verbal fluency was found. ${ }^{8}$ Accordingly, our result can help broaden our knowledge about the interaction between the APOE $\varepsilon 4$ allele and TSH significantly affecting SM or even executive components embedded in VFT at least across $\mathrm{CN}$ elderly population.

While our study illustrated the interaction between the APOE $\varepsilon 4$ allele and TSH appeared to interfere with SM performance in comparison with EM tests among $\mathrm{CN}$ elderly subjects, several limitations can be noticed.

First of all, as previously mentioned, the score of VFT could be interpreted as the combination of SM and executive function. Consequently, our findings may not ensure whether the influence of the interaction on VFT was confined to SM or executive abilities. Even if the interaction influenced both factors, our outcomes did not still clarify to what extent semantic component was influenced by this interaction. The neuropsychological test, CERAD-K, used in the study contains two types of Trail Making Tests (TMTs) designed to measure an executive compartment of cognitive function. The analysis of our results, however, did not include these tests, since a large proportion 
of participants were not able to complete TMTs, especially a part B of TMT (TMB) which requires subjects to connect numbers and consonant letters in an alternating sequence. The average year of education of elderly participants (6.81 years) may account for this failure to accomplish TMB, which is supported by one study showing the correlation between age, an education level and the performance of TMT among Korean elderly population. ${ }^{40}$ This drawback could be addressed by a future study including additional tests measuring executive function.

Apart from this, this study depended on CN elderly participants residing in the rural area and the mean value of their educational level recorded 6.81, which was below the 9-yearperiod of mandatory education in Korea. Therefore, further studies recruiting more heterogeneous groups in terms of age, cognitive function, such as, those with $\mathrm{MCI}$ and $\mathrm{AD}$, as well as geometrical and educational parameters, could be conducted to ensure if our findings can be replicated. This may help address compounding factors embedded in characteristics of subjects in our study.

In light of biological markers, TSH and $\mathrm{fT} 4$ were employed as representatives of thyroid function in this study. Other markers, such as triiodothyronine and thyroid-related antibody titers, were not taken into consideration, so that further studies considering diverse thyroid-related indicators would provide clearer elucidation concerning the interplay between thyroid function and APOE. Moreover, the analysis of APOE in our study did not consider homozygous and heterozygous types of the $\varepsilon 4$ allele, indicating that a dosing effect related to APOE could not be identified and thereby future research will be needed to manage this aspect.

In conclusion, despite the limitations embedded in this research, our findings have demonstrated the significant interaction between the APOE $\varepsilon 4$ allele and TSH could exist in relation to the impaired performance in SM among CN elderly. Accumulated data have suggested possibilities of the association between the APOE $\varepsilon 4$ allele, thyroid function and cognitive performance, although discrepancies exist among investigations and exact mechanisms behind this association has not been elucidated. Impaired or diminished declarative memory is regarded as one of early symptoms in $\mathrm{AD}$ and the presence of the APOE $\varepsilon 4$ allele is a risk factor of $\mathrm{AD}$ as well. Incorporating with the fact that aging accompanies the alteration of thyroid function, the complex interplay between thyroid-related parameters and the APOE polymorphism related to cognitive abilities requires further studies.

\section{Acknowledgments}

This research was supported by Basic Science Research Program through the National Research Foundation of Korea (NRF) funded by the Ministry of Education (NRF-2019R1I1A3A01058477). This research was partially supported by the National Research Foundation of Korea (NRF) grant funded by the Korea government (MSIT) (NRF-2017R1A2B4005223).

\section{Conflicts of Interest}

The authors have no potential conflicts of interest to disclose.

\section{Author Contributions}

Conceptualization: Seok Woo Moon. Data curation: Beomjun Kim. Formal analysis: Seok Woo Moon. Funding acquisition: Seok Woo Moon. Investigation: Beomjun Kim, Seok Woo Moon. Methodology: Seok Woo Moon. Project administration: Beomjun Kim, Seok Woo Moon. Resource: Seok Woo Moon. Software: Beomjun Kim. Supervision: Seok Woo Moon. Validation: Beomjun Kim, Seok Woo Moon. Visualization: Beomjun Kim. Writing_original draft: Beomjun Kim. Writing_review\&editing: Beomjun Kim, Seok Woo Moon.

\section{ORCID iDs}

Beomjun Kim

Seok Woo Moon https://orcid.org/0000-0002-1074-8122

\section{REFERENCES}

1. Rasmussen J, Langerman H. Alzheimer's disease - Why we need early diagnosis. Degener Neurol Neuromuscul Dis 2019;9:123-130.

2. Dubois B, Picard G, Sarazin M. Early detection of Alzheimer's disease: new diagnostic criteria. Dialogues Clin Neurosci 2009;11:135-139.

3. Yiannopoulou KG, Papageorgiou SG. Current and future treatments for Alzheimer's disease. Ther Adv Neurol Disord 2013;6:19-33.

4. Galimberti D, Scarpini E. Disease-modifying treatments for Alzheimer's disease. Ther Adv Neurol Disord 2011;4:203-216.

5. Breteler MM, van Duijn CM, Chandra V, Fratiglioni L, Graves AB, Heyman A, et al. Medical history and the risk of Alzheimer's disease: a collaborative re-analysis of case-control studies. EURODEM Risk Factors Research Group. Int J Epidemiol 1991;20 Suppl 2:S36-S42.

6. Ganguli M, Burmeister LA, Seaberg EC, Belle S, DeKosky ST. Association between dementia and elevated TSH: a community-based study. Biol Psychiatry 1996;40:714-725.

7. Martins CA, Oulhaj A, de Jager CA, Williams JH. APOE alleles predict the rate of cognitive decline in Alzheimer disease: a nonlinear model. Neurology 2005;65:1888-1893.

8. Reas ET, Laughlin GA, Bergstrom J, Kritz-Silverstein D, Barrett-Connor E, McEvoy LK. Effects of APOE on cognitive aging in communitydwelling older adults. Neuropsychology 2019;33:406-416.

9. Bature F, Guinn BA, Pang D, Pappas Y. Signs and symptoms preceding the diagnosis of Alzheimer's disease: a systematic scoping review of literature from 1937 to 2016. BMJ Open 2017;7:e015746.

10. Delange F. Iodine deficiency as a cause of brain damage. Postgrad Med J 2001;77:217-220.

11. Thompson CC, Potter GB. Thyroid hormone action in neural development. Cereb Cortex 2000;10:939-945.

12. Rivas M, Naranjo JR. Thyroid hormones, learning and memory. Genes Brain Behav 2007;6 Suppl 1:40-44.

13. Koibuchi N. Hormonal regulation of cerebellar development and plasticity. Cerebellum 2008;7:1-3.

14. Annerbo S, Wahlund LO, Lokk J. The significance of thyroid-stimulating hormone and homocysteine in the development of Alzheimer's disease in mild cognitive impairment: a 6-year follow-up study. Am J Alzheimers Dis Other Demen 2006;21:182-188.

15. Annerbo S, Lokk J. A clinical review of the association of thyroid stimulating hormone and cognitive impairment. ISRN Endocrinol 2013; 2013:856017.

16. Resta F, Triggiani V, Barile G, Benigno M, Suppressa P, Giagulli VA, et al. Subclinical hypothyroidism and cognitive dysfunction in the elderly. Endocr Metab Immune Disord Drug Targets 2012;12:260-267.

17. Hu Y, Wang ZC, Guo QH, Cheng W, Chen YW. Is thyroid status asso- 
ciated with cognitive impairment in elderly patients in China? BMC Endocr Disord 2016;16:11.

18. Parsaik AK, Singh B, Roberts RO, Pankratz S, Edwards KK, Geda YE, et al. Hypothyroidism and risk of mild cognitive impairment in elderly persons: a population-based study. JAMA Neurol 2014;71:201-207.

19. de Jongh RT, Lips P, van Schoor NM, Rijs KJ, Deeg DJ, Comijs HC, et al. Endogenous subclinical thyroid disorders, physical and cognitive function, depression, and mortality in older individuals. Eur J Endocrinol 2011;165:545-554.

20. Grigorova M, Sherwin BB. Thyroid hormones and cognitive functioning in healthy, euthyroid women: a correlational study. Horm Behav 2012;61:617-622.

21. Bojar I, Owoc A, Gujski M, Witczak M, Gnatowski M, Walecka I. Functional status of thyroid and cognitive functions after menopause. Med Sci Monit 2015;21:1625-1633.

22. Wahlin A, Bunce D, Wahlin TB. Longitudinal evidence of the impact of normal thyroid stimulating hormone variations on cognitive functioning in very old age. Psychoneuroendocrinology 2005;30:625-637.

23. Wisdom NM, Callahan JL, Hawkins KA. The effects of apolipoprotein $\mathrm{E}$ on non-impaired cognitive functioning: a meta-analysis. Neurobiol Aging 2011;32:63-74.

24. Hirono N, Hashimoto M, Yasuda M, Kazui H, Mori E. Accelerated memory decline in Alzheimer's disease with apolipoprotein epsilon4 allele. J Neuropsychiatry Clin Neurosci 2003;15:354-358.

25. Nilsson LG, Adolfsson R, Backman L, Cruts M, Nyberg L, Small BJ, et al. The influence of APOE status on episodic and semantic memory: data from a population-based study. Neuropsychology 2006;20:645-657.

26. Kim KW, Jhoo JH, Lee KU, Lee DY, Lee JH, Youn JY, et al. Association between apolipoprotein E polymorphism and Alzheimer's disease in Koreans. Neurosci Lett 1999;277:145-148.

27. Lee DY, Lee KU, Lee JH, Kim KW, Jhoo JH, Kim SY, et al. A normative study of the CERAD neuropsychological assessment battery in the Korean elderly. J Int Neuropsychol Soc 2004;10:72-81.

28. Kalmijn S, Mehta KM, Pols HA, Hofman A, Drexhage HA Breteler MM. Subclinical hyperthyroidism and the risk of dementia. The Rotterdam study. Clin Endocrinol (Oxf) 2000;53:733-737.

29. Ceresini G, Lauretani F, Maggio M, Ceda GP, Morganti S, Usberti E, et al. Thyroid function abnormalities and cognitive impairment in elderly people: results of the Invecchiare in Chianti study. J Am Geriatr Soc 2009;57:89-93.

30. Dezonne RS, Lima FR, Trentin AG, Gomes FC. Thyroid hormone and astroglia: endocrine control of the neural environment. J Neuroendocrinol 2015;27:435-445.

31. Trentin AG. Thyroid hormone and astrocyte morphogenesis. J Endocrinol 2006;189:189-197.

32. Nomoto S, Kinno R, Ochiai H, Kubota S, Mori Y, Futamura A, et al. The relationship between thyroid function and cerebral blood flow in mild cognitive impairment and Alzheimer's disease. PLoS One 2019; 14:e0214676.

33. Brewster PW, Melrose RJ, Marquine MJ, Johnson JK, Napoles A, MacKay-Brandt $\mathrm{A}$, et al. Life experience and demographic influences on cognitive function in older adults. Neuropsychology 2014;28:846-858.

34. Eich TS, Tsapanou A, Stern Y. When time's arrow doesn't bend: APOEepsilon 4 influences episodic memory before old age. Neuropsychologia 2019;133:107180.

35. Bayles KA, Tomoeda CK, Kaszniak AW, Trosset MW. Alzheimer's disease effects on semantic memory: loss of structure or impaired processing? J Cogn Neurosci 1991;3:166-182.

36. Bojar I, Stasiak M, Cyniak-Magierska A, Raczkiewicz D, Lewinski A. Cognitive function, APOE gene polymorphisms, and thyroid status associations in postmenopausal women in Poland. Dement Geriatr Cogn Disord 2016;42:169-185.

37. Roman C, Fuior EV, Trusca VG, Kardassis D, Simionescu M, Gafencu $\mathrm{AV}$. Thyroid hormones upregulate apolipoprotein $\mathrm{E}$ gene expression in astrocytes. Biochem Biophys Res Commun 2015;468:190-195.

38. Nyberg L, Backman L, Erngrund K, Olofsson U, Nilsson LG. Age differences in episodic memory, semantic memory, and priming: relationships to demographic, intellectual, and biological factors. J Gerontol B Psychol Sci Soc Sci 1996;51:P234-P240.

39. Henry JD, Crawford JR, Phillips LH. Verbal fluency performance in dementia of the Alzheimer's type: a meta-analysis. Neuropsychologia 2004;42:1212-1222.

40. Seo EH, Lee DY, Kim KW, Lee JH, Jhoo JH, Youn JC, et al. A normative study of the Trail Making Test in Korean elders. Int J Geriatr Psychiatry 2006;21:844-852. 